\title{
Universality of the Fluctuations of Growing Interfaces
}

\#1. Growing interfaces uncover universal fluctuations behind scale invariance

Authors: Kazumasa A. Takeuchi, Masaki Sano, Tomohiro Sasamoto and Herbert Spohn

Scientific Reports 1, 34 (2011)

\#2. Universal fluctuations of growing interfaces: evidence in turbulent liquid crystals

Authors: Kazumasa A. Takeuchi and Masaki Sano

Phys. Rev. Lett. 104, 230601 (2010)

\#3. One-dimensional Kardar-Parisi-Zhang equation: an exact solution and its universality

Authors: Tomohiro Sasamoto and Herbert Spohn

Phys. Rev. Lett. 104, 230602 (2010)

\section{Recommended with a commentary by Mehran Kardar, MIT}

The scale invariant fluctuations of a growing interface represent a basic problem in non-equilibrium statistical physics. The KPZ equation, introduced in 1986, provided a framework and perspective for describing universality in this problem. Surprising connections of the KPZ equation to other interesting problems, such as fluctuations of a line pinned by impurities, and to Burgers turbulence, spawned subsequent intense activity in this topic. On the theoretical front various scaling laws and universality classes were identified, but a complete characterization of scaling functions was left missing. While a host of numerical simulations confirmed expected scaling laws, a number of experimental attempts at verification were at best inconclusive (likely due to presence of features such as quenched disorder and long-range interactions). Remarkably, in the last year there have been major advancement on both these fronts, as succinctly described in the review by Kazumasa et. al. (\#1).

The experiments reported examine the interface between two turbulent states (DSM1 and DSM2) of a nematic liquid crystal, presented initially in the article above by Takeuchi and Sano (\#2). The liquid crystal phase DSM2 can be nucleated with an ultraviolet laser pulse and grows into DSM1 at high applied electric fields. The nucleation can be at a point leading to circular growth, or along a line, leading to planar growth. (The liquid crystal is confined in a thin container resulting in a two dimensional setup.) The growing cluster is photographed and its fluctuations are then characterized. The experimental system appears to be free of the difficulties that marred previous experiments as the scaling exponents of the KPZ equation are observed. More convincingly, the copious data from experiments provide the distribution function of height fluctuations in agreement with theory (below).

The theoretical advance is based on yet another unexpected connection, this time to random matrix theory. Tracy and Widom characterized the fluctuations of the largest eigenvalue of an $N x N$ matrix[1]. In paper \#3 above Sasamoto and Spohn demonstrate that height fluctuations of the KPZ interface are also characterized by 
this Tracy-Widom distribution. This coincided with a host of independent activity last year demonstrating the connection of the KPZ model to the Tracy-Widom distribution via Bethe Ansatz analysis of the replicated directed polymer problem[2, $3,4]$.

Paper \#1 synthesizes a number of experimental and theoretical results. In particular, it notes that the height fluctuations for circular growth (nucleation from a point) are distinct from those of planar growth (starting from a line): the former is described by the Gaussian Unitary Ensemble (GUE) of random matrices, the latter by the Gaussian Orthogonal Ensemble (GOE).

\section{References}

[1] Level-spacing distributions and the Airy kernel, C. Tracy and H. Widom, Commun. Math. Phys. 159, 151 (1994); On orthogonal and symplectic matrix ensembles, C. Tracy and H. Widom, Commun. Math. Phys. 177, 727 (1996).

[2] Bethe ansatz derivation of the Tracy-Widom distribution for one-dimensional directed polymers, V. Dotsenko, Europhys. Lett. 90, 20?003 (2010).

[3] Intermediate Disorder Regime for Directed Polymers in Dimension 1+1, T. Alberts, K. Khanin, and J. Quastel, Phys. Rev. Lett. 105, 090603 (2010).

[4] Exact Solution for the Kardar-Parisi-Zhang Equation with Flat Initial Conditions, P. Calabrese and P. Le Doussal, Phys. Rev. Lett. 106, 250603 (2011). 